\title{
Light is Loud \\ A Sound Driven LED Suit
}

\author{
Artur Aguiar \\ Virginia Tech \\ Blacksburg, VA, USA \\ arturma@vt.edu
}

\author{
Bryan Malyn \\ Virginia Tech \\ Blacksburg, VA, USA \\ bmalyn@vt.edu \\ Steve Harrison \\ Virginia Tech \\ Blacksburg, VA, USA \\ srh@cs.vt.edu
}

\author{
Evan Lobeto \\ Virginia Tech \\ Blacksburg, VA, USA \\ elobeto@vt.edu
}

\begin{abstract}
We approach performance as fundamentally a hybrid situation: that performer and technology are united in a postphenomenological embrace. Light is Loud takes this as a starting point: the form of the performer is subsumed into an array of lights that takes the temporal shape of a selfreferential text. The loss of the human form in the piece becomes a critical statement on the ambiguity of hybridity.
\end{abstract}

In a completely darkened space, a figure with some strips of LEDs speaks a short poem overtly on the nature of "loud". While the title of the piece, Light is Loud, suggests "dazzling", the effect is a meditation on the nature of quiet.

\section{ACM Classification Keywords}

A.0 GENERAL: Conference proceedings; H.5.1 Multimedia Information Systems: Audio input/output; H.5.2 User Interfaces: Auditory (non-speech) feedback

\section{Author Keywords}

Audio Processing; Synesthesia in Art; Spoken Word

Performance; Demos

\section{INTRODUCTION}

Our LED suit is made up of strips of LEDs which crisscross the body (see Figure 1). Unlike other LED suits on the market ours reacts dynamically to the the sounds in the environment.

The LED strips are powered by 4 AA batteries, and controlled by an Arduino Mega (see Figure 2). There are two pushbuttons which allow us to move, forwards and backwards, through the three modes that we have. The first mode stores the value of accumulated amplitudes above a threshold, while the second and third modes use the average frequency from a 8 bit Fixed Fast Fourier Transform (FFT)[?]. These characteristics are mapped to a temperature from $1.000 \mathrm{~K}$ to 15.000 $\mathrm{K}$, from which the colour temperature is then calculated[?].

Copyright 2015 is held by the author(s). Publication rights licensed to Aarhus University and ACM.

5th Decennial Aarhus Conference on Critical Alternatives August 17 21, 2015, Aarhus Denmark

DOI: http://dx.doi.org/10.7146/aahcc.v1i1.21320

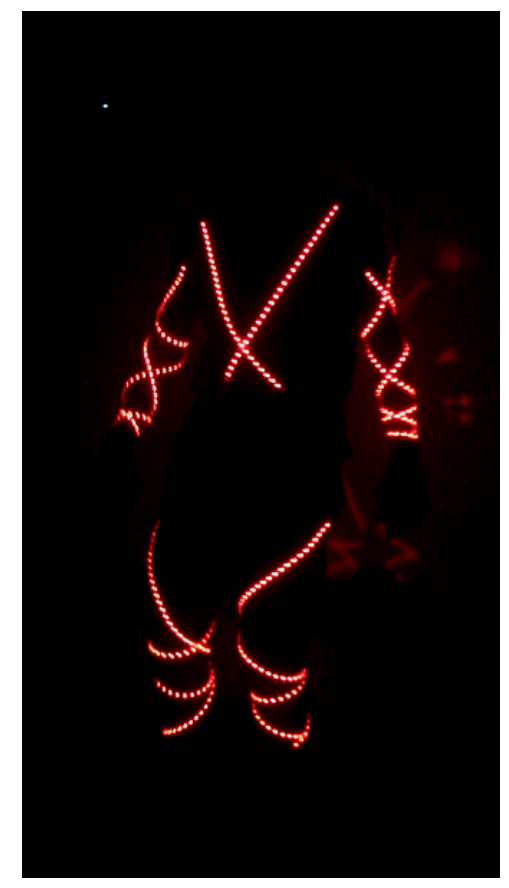

Figure 1. LED Suit

The first mode starts off with all of the lights being set to a dim red. When the amplitude rises above the specified threshold the lights get brighter and move towards a blue hue. In the second mode, when the amplitude of sound is above the threshold, LEDs at the shoulders turn on and then flow down the entire body. The third mode works like an equalizer, mirrored around the waist, with the amplitude of sound controlling how many of the LEDs are on.

\section{TECHNICAL REQUIREMENTS}

For our demo we need a dark room, a wireless microphone (preferably hands-free), and an $3.5 \mathrm{~mm}$ auxiliary connection so that we can play sounds over the speakers in the room.

VIDEO

http://youtu.be/ZNu3zdZL3wE 


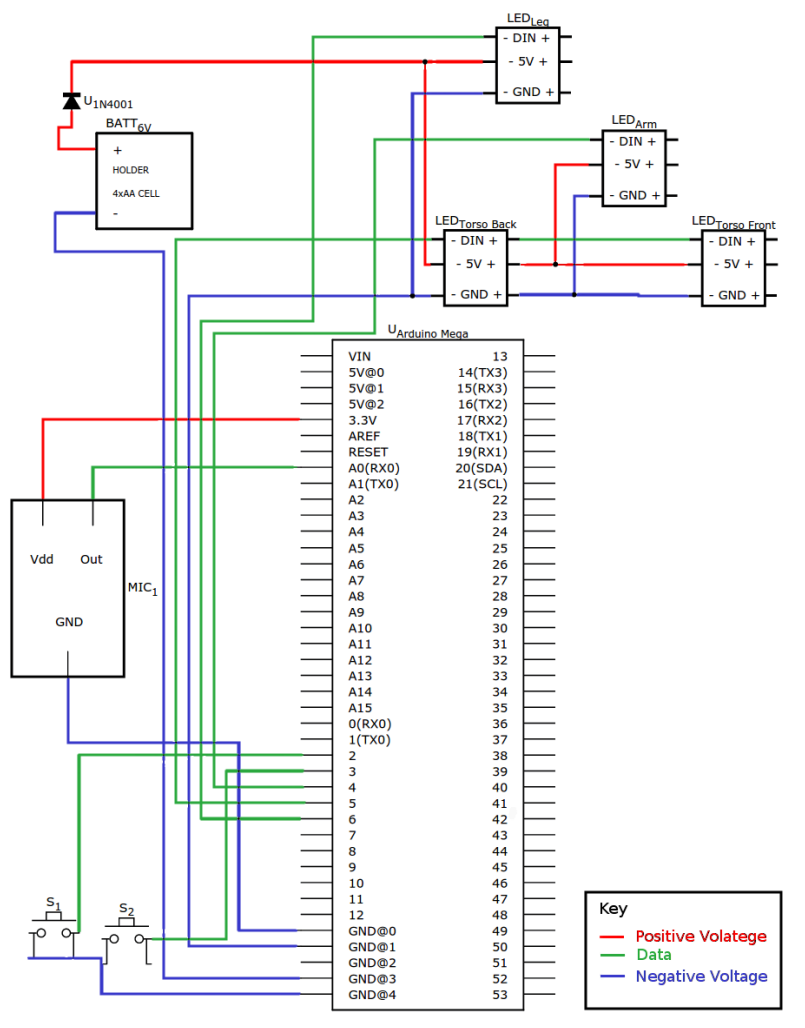

Figure 2. LED Suit Schematic

\section{SYNESTHESIA IN ART}

There is a wide spectrum of synesthesia in art, both by synesthetes, based off of what they experience as synesthetes; and by non-synesthetes, which aim at giving a general audience a synesthetic-like experience. This project falls into the latter camp.

The overall effect of the LED suit is a simulation of soundcolour synesthesia, or chromesthesia; a type of synesthesia in which heard sounds automatically and involuntarily evoke an experience of colour[?]. Individuals with sound-colour synesthesia are consciously aware of their synesthetic colour associations/perceptions in daily life. That is, the synesthetic colour experience supplements, but does not obscure real, modality-specific perceptions.[?]

Our project uses a sound to colour model based on the heat of the sound in the environment. The three modes are designed to take the same information, the sound in the environment, and give different visual representations of that data: the first mode is a visualisation of the build up of sound; the second mode gives the sound the appearance of being a fluid, pouring down the body; and the third mode shows the amplitude of sound, as though the wearer were an equalizer.

\section{"LIGHT IS MY ADDICTION"}

Light is Loud is a spoken word performance based on two poems: Endless Noise by Nandita Das and The Duality Of Darkness And Light by Tim Vallie. The LED technology (see Figure 1) is symmetrically arranged on the limbs of the per- former. The design emphasizes the spatiality, rather than the human form underneath. That is, the human disappears into the bands of light. The performer's motions are direct, but unexpectedly, not rhythmic or easily interpreted as expressive. The effect is intentionally ambiguous.

The poem is in the first-person, heightening the ambiguity: is it the person or the technology that is speaking?

We see the on-going shift of our general relation to technology - that is, from hybridity to the organization of human activity - echoing in the performance. The performer becomes a kind of "hybrid", bound by the lights. Alternatively, the arrangements of lights become a replacement for the human form.

The canonical human relation to media technology is a kind of entangled embodied experience: whether it is exercising to pop music with a set of headphones or loosing oneself in a video game, bodily interaction is in some sense externally synced to the technology. Light is Loud takes a critical stance by inverting that relation, while remaining entangled and embodied: the motion of the LED strips is tied to bodily motion and the expression of the light pattern tied to the utterances of the wearer.

Many may see the piece initially as a caricature of a toreador's "suite of lights". Indeed, the performer moves about as though strutting amongst the bulls in the ring. But that is a misleading reading since the toreador is responding to a dangerous "other", where the performer here is forming space through the moving LED strips, around the stage.

\section{CONCLUSION}

This work can be appreciated from many different perspectives, such as synesthesia in art or as a futuristic, Tron-like, article of clothing. The concepts used in this project could be used to map attributes of the person wearing the suit or the environment around them. An example of this could be to use the motion of the wearer's limbs to glow different colours based off of orientation and/or the speed at which they are moving. The number of possible combinations of inputs and their associated impacts makes the mind boggle. 